\title{
Funcionamiento de las criptomonedas de menos de 1USD Una revisión literaria
}

\author{
How cryptocurrencies under 1USD work: A literature review
}

Como funcionam as criptomoedas abaixo de US \$ 1: uma revisão literária

\section{ARTÍCULO GENERAL}

\section{César Loo Gil \\ cesarloo@,biofab.com.pe \\ https://orcid.org/0000-0001-8396-5972}

Científico Investigador de BioFab Inc.y del Centro de Investigación \& Producción Científica IDEOs, Lima - Perú

Recibido 07 de Abril 2021 | Arbitrado y aceptado 19 de Junio 2021 | Publicado en 03 Setiembre 2021

\section{RESUMEN}

Este artículo se centra en una metodología de estudio que interrelaciona elementos de la gestión de la información con aspectos de la investigación de operaciones (más precisamente cadenas de Markov y teoría de colas), con la intención de demostrar, en un caso concreto (el uso de la criptomoneda bitcoin), de qué manera ambas técnicas pueden combinarse, a partir de la aplicación conjunta de elementos asociados al análisis cuantitativo y cualitativo, para caracterizar un objeto o proceso, capturar datos e información asociados a este, predecir sus comportamientos y transformar dichos elementos informacionales en valor agregado que conlleve a una acertada toma de decisiones, en beneficio de un usuario y su contexto.

Palabras clave: criptomoneda bitcoin, gestión de información, investigación de operaciones, valor agregado.

\section{ABSTRACT}

This article focuses on a study methodology that interrelates elements of information management with aspects of operations research (more precisely Markov chains and queuing theory), with the intention of demonstrating, in a specific case (the use of the cryptocurrency bitcoin), how both techniques can be combined, from the joint application of elements associated with quantitative and qualitative analysis, to characterize an object or process, capture data and information associated with it, predict its behavior and transform said informational elements in added value that lead to a successful decision-making, for the benefit of a user and their context.

Keywords: bitcoin cryptocurrency, information management, operations research, added value.

\section{RESUMO}

Este artigo enfoca uma metodologia de estudo que interrelaciona elementos da gestão da informação com aspectos da pesquisa operacional (mais precisamente cadeias de Markov e teoria das filas), com o intuito de demonstrar, em um caso específico (o uso da criptomoeda bitcoin), como ambas as técnicas podem ser combinados, a partir da aplicação conjunta de elementos associados à análise quantitativa e qualitativa, para caracterizar um objeto ou processo, captar dados e informações a ele associados, prever seu comportamento e transformar esses elementos informativos em valor agregado que conduzam a uma decisão bemsucedida. fazer, para o benefício de um usuário e seu contexto.

Palavras-chave: criptomoeda bitcoin, gestão da informação, pesquisa operacional, valor agregado. 


\section{Introducción}

El dinero debe ser una de las creaciones humanas más exitosas en términos de extensión y masividad. Sus formas, sin embargo, son múltiples. Por ejemplo, hoy presenciamos un boom de criptomonedas que amenaza con dejar obsoleto al dinero físico (Baraona \& Reyes, 2018).

En el marco de la sociedad de la información, bajo el cual se desenvuelve, hoy día, el ser humano y que corresponde a un mundo supremamente globalizado (Escobar-Sarria, 2014; Ziemba, 2019), la búsqueda de oportunidades de desarrollo con base en la gestión de la información se convierte en una tarea importante.

Dentro de este contexto, existe un marcado interés por parte de muchas personas por encontrar elementos diferenciales que les permitan obtener los mejores dividendos ante esta rea- lidad, razón por la cual, la generación de valor con base en dicha información se convierte en un afán innegable, máxime si este puede otorgarles dividendos, muchos de ellos econó- micos, significativos (Vásquez-Rizo \& Gabalán-Coello, 2017).

Es así como ha surgido y está tomando mucha fuerza el mercado emergente de las criptomonedas (Oliveira-Monteiro, De Souza, Guazzelli-Batista, \& Zaparoli, 2019), el cual cuenta con una amplia variedad de criptoactivos (entre ellos, la información), los cuales generan continuamente oportunidades de inversión y posibilidades de impensada prosperidad en la sociedad moderna, debido a sus constantes mo- vimientos y fluctuación, así como a la información que nutre dicho mercado y que este, a su vez, genera.

Por tal razón, este contexto se convierte en un escenario interesante de análisis que puede ser estudiado desde distintas perspectivas académicas, entre ellas, la gestión de la información, desde un punto de vista cualitativo (ciencias sociales), junto con la investigación de operaciones, desde una óptica cuantitativa (ingeniería), con la intención de buscar una si- nergia entre ambos componentes. Una articulación bajo esta misma lógica la proponen Capps, Cassidy, Gravois y Warner (2019), la cual permite enten- der y estudiar dicha temática desde dos orillas que parecieran distantes, pero que pueden complementarse, según el estudio que aquí se desea.

De esta manera, el presente artículo utiliza la teoría de las cadenas de Markov para hallar dicha sinergia y para guiar a los sujetos inmersos en la sociedad de la información hacia una posibilidad de obtención de valor agregado relativamente nueva, en la que puedan encontrar, a través de la recopilación de información del mer- cado de las criptomonedas (la más representativa: bitcoin), una opción viable de manejo de datos y de información —y hasta de inversión-; en la cual, además, entiendan los riesgos y los beneficios que 
puede otorgarles dicho criptoactivo, tan poco analizado (por lo menos de esta forma), pero tan influyente en el mundo globalizado.

\section{Metodología}

La búsqueda de la información para la revisión de literatura se hizo consultando las siguientes bases de datos como son Proquest, Scopus y Google Scholar para encontrar revistas del más alto nivel científico. Asimismo, cabe mencionar que para la realización de lo mencionado se emplearon también palabras clave como como Criptomonedas, Dinero Electrónico, Dólar Americano y otros relacionados a tipos de criptomonedas específicos.

Cada uno de estos descriptores se combinaron entre sí durante la búsqueda utilizando los operadores boléanos "and" y “or". Además, algunos de los criterios o filtros que se utilizaron para hacer muchos más específica la búsqueda y ser más precisos con los documentos encontrados en las diferentes bases de datos consultadas fueron: que sean artículos de revistas, y la antigüedad de la publicación no mayor a 10 años, procurando que la mayor parte de las fuentes se mantenga en 5 años atrás.

Toda la información previa relacionada a los filtros aplicados y las especificaciones sirvieron como criterios de inclusión, es decir que sean artículos publicados en revistas científicas, que no excedan los cinco años de antigüedad, que la temática guarde relación con las variables de Criptomonedas y con un valor de menos de 1 USD. Se excluyeron, en tanto, todos los documentos que estuvieran orientados a otro tipo de criptomonedas.

\section{Resultados y discusión}

\subsection{Aspectos tecnológicos}

Las criptomonedas se emiten al margen de los gobiernos y bancos centrales y, al menos en teoría, esta función se traslada a todo aquel que quiera participar. Esta generación de moneda se denomina "minado". Estos participantes (mineros) son quienes aportan la seguridad a las transacciones utilizando, en la mayor parte de los casos, la tecnología de blockchain (cadena de bloques).

Blockchain es un gigantesco libro de contabilidad distribuido (DLT) en el que los registros (bloques) están enlazados para proteger la seguridad y la privacidad de las transacciones. Se trata de una base de datos distribuida y segura gracias a la utilización de algoritmos criptográficos.

Al realizar una transacción hay varios usuarios (nodos) que se encargan de verificar la transacción y registrarlas en el gigantesco libro de cuentas. Veamos el funcionamiento 
con un ejemplo. Imaginemos que A quiere enviar a B una determinada cantidad X. Si esta transacción se realizara mediante una transferencia bancaria, A le pediría a su banco que retirara $\mathrm{X}$ de su cuenta para enviarla a la cuenta de $\mathrm{B}$. Lo restarían de su saldo, comunicando al otro banco que debe añadir X a la cuenta de B.

Pues bien, esta misma transacción realizada con criptomonedas, tal y como se representa en el en el siguiente gráfico elaborado específicamente para este trabajo, funcionaría de esta forma. La primera diferencia sustancial es que nadie sabrá quién es A ni B, únicamente que desde un monedero digital (equivalente a la cuenta bancaria) se quiere transferir una cantidad a otro monedero. A envía un mensaje a los usuarios notificando su intención. Estos comprueban que tiene saldo suficiente. Si es así, anotan esta transacción de forma provisional. Conforme pasa el tiempo y se van completando más transacciones se va conformando un bloque. Al llegar a la capacidad de la cadena de bloques se procede a validarlo.

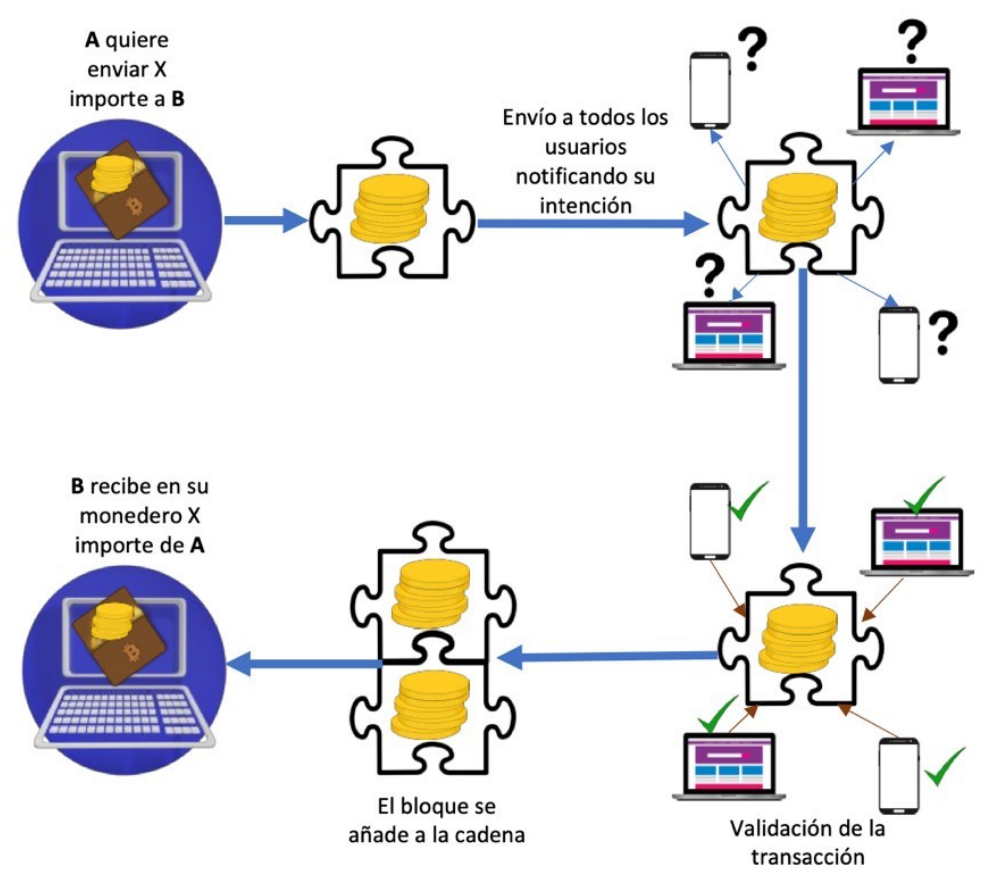

Mediante complicados algoritmos matemáticos que requieren elevada potencia de computación, y por lo tanto alto consumo energético, los bloques quedan registrados de forma permanente en la cadena. Uno solo de estos bloques no podría ser modificado sin alterar todos los que están enlazados con él, algo que sería realmente improbable pues el resto de los nodos lo deberían validar. Este mecanismo asegura la integridad de la operación. 
En el proceso de minado, los mineros reciben avisos de nuevas transacciones agrupadas en bloques. Unos compiten con otros y el primero que consigue crear un bloque válido recibe una recompensa por el servicio, en la criptomoneda que esté operando.

La cadena de bloques está sincronizada entre los nodos de forma irreversible. Nadie podrá modificar el libro de registro sin que el resto se entere.

Esta tecnología permite asimismo asegurar la trazabilidad de las transacciones. Aunque no sean conocidas las identidades de $\mathrm{A}$ y $\mathrm{B}$, es público el camino que ha seguido el envío.La transferencia de $\mathrm{A}$ a $\mathrm{B}$ se realiza de forma rápida y sin comisiones.

\subsection{Criptomonedas}

La historia de las criptomonedas comienza necesariamente con el Bitcoin y la tecnología Blockchain, punto de partida para el origen de la mayor parte de las criptomonedas que hoy superan las 2,000 referencias disponibles en el mercado (Jimenez \& Contreras, 2018).

\section{Ventajas}

Sustancialmente más baratas y rápidas que las realizadas por las redes de pago convencionales, al no tener que pagar más comisión que la mínima que existe en la actualidad como contraprestación que se otorga a los mineros por comprobar el "doble gasto en la cadena blockchain (Onrubia, 2017)

Vaya por delante que es indudable que la criptomoneda más conocida es el bitcóin, si bien puede sorprender el número de ellas existente. En www.investing.com están contabilizadas a fecha de cierre del presente trabajo (mayo de 2019), más de dos mil quinientas. Bien es verdad que se suele hablar de que están operando unas mil quinientas. Para comprender el alcance de las definiciones que se aportan, y, sobre todo, la finalidad que, en algún caso, se desea imprimir, resulta necesario identificar las principales utilidades que tienen (o no, dependiendo de quién emita la opinión), las monedas virtuales. Son coincidentes -o pueden serlo, dependiendo, reitero, de quién emita su parecer- con las del dinero: depósito de valor (transfiere el poder de compra del presente al futuro, de modo que quien recibe una criptomoneda como contraprestación por el objeto vendido puede, a su vez, utilizarla para comprar, sin que sea necesario que conserve exactamente el mismo valor), unidad de cuenta (permite fijar y conocer el precio de lo que se quiere comprar o vender), y medio de pago o de cambio (permite realizar cualquier tipo de transacción, facilitando el intercambio de bienes y servicios, de suerte que es aceptada como contraprestación en la compraventa) 
Resulta obvio que el hecho de que las criptomonedas cumplan, o puedan cum- plir, las funciones que se le atribuyen al dinero en términos económico-financieros, no implica, por sí mismo, que tengan, o deban tener, tal consideración desde un punto de vista jurídico. Tal como tendremos ocasión de comprobar, la existencia de regulaciones nacionales no ha ido acompañada de su reconocimiento como monedas fiduciarias. En el mejor de los casos, se le ha reconocido legalmente alguna de las utilidades apuntadas.

Expongo las definiciones de bitcóin porque, de entrada, no es correcto reflexio- nar sobre algo novedoso sin al menos describirlo. Me parece relevante advertir, sin embargo, que gran parte del debate gira en torno a qué son las criptomonedas, en general, y el bitcóin, en particular; y que esa controversia no tiene una especial trascendencia desde el punto de vista jurídico-penal; o, al menos, no tiene el alcance que los economistas y otros le confieren10. Lo relevante para nosotros no es lo que sea, sino que, lo que sea, se está empleando para la comisión de hechos delicti- vos graves, como es el blanqueo de dinero (o la financiación del terrorismo, el narcotráfico, o la defraudación tributaria), cuya comisión no depende de la naturaleza jurídica del medio empleado -más allá, obviamente, de que reúna unas cuali- dades mínimas.

Con ello tampoco estoy negando que ese debate pueda tener interés, incluso para nosotros. Pienso, por ejemplo, en el decomiso. Queda al margen, además, su interés en sede penal cuando es el objeto material del delito, pues de su naturaleza jurídica dependerá que el juicio de tipicidad sobre determinadas conductas haya que ubicar- lo en un delito u otro. En otro orden de cosas, tal como apunta acertadamente el BCE en su informe sobre monedas virtuales de febrero de 2015, la definición de ellas tiende a depender del contexto, de suerte que una perspectiva antiblanqueo, $\mathrm{u}$ otra que ponga el acento en aspectos relativos al registro y licencia de los intervinientes en el mercado virtual, tendrá reflejo en la definición.

Naturalmente, a lo anterior hay que sumar las lógicas revisiones de posiciones iniciales acordes con la evolución - por razones de diversa consideración del concepto a definir13. Así, sostiene el BCE que su definición aportada en 2012 ("Una moneda virtual es un tipo de dinero digital no regulado, que es emitido y generalmente controlado por sus desarrolladores, y utilizado y aceptado entre los miembros de una comunidad virtual específica") debe ser revisada por tres razones:

Primera. Ya no debe contener la palabra "dinero", pues ha quedado claro que, incluso hoy, las monedas virtuales no tienen la naturaleza de un activo muy líquido, y no han alcanzado el nivel de aceptación comúnmente asociado con el dinero. 
Segunda. La palabra "no regulado" debería suprimirse de la definición utilizada en 2012, dado que, en algunas jurisdicciones, la legislación y la reglamentación se han adaptado a esta innovación, además de abordar algunos de sus aspectos o cuestiones de los servicios conexos.

Tercera. Para evitar malos entendidos respecto a cualquier límite teórico a la aceptación de una moneda virtual, también se elimina "utilizada y aceptada entre los miembros de una comunidad virtual específica", aunque las redes peer-to-peer e Internet pueden ser descritas como una comunidad virtual.

Resultado de todo lo anterior, el BCE define ahora la moneda virtual como "una representación digital de valor, no emitida por ninguna autoridad bancaria central, institución de crédito o emisor de dinero electrónico reconocido, que, en ciertas ocasiones, puede ser utilizada como medio de pago alternativo al dinero". Intenta alejarse, como puede observarse, de una concepción neta de dinero.

Una de las principales razones para proceder a su tratamiento y regulación es, como he dicho, evitar su uso delictivo, destacadamente, en el ámbito del blanqueo de dinero y de la financiación del terrorismo. Desde esta perspectiva se explica perfectamente que la nueva Directiva de la Unión Europea, dedicada a la lucha contra estos dos graves fenómenos criminales, le preste su atención, e inste, precisamente, a la regulación por las jurisdicciones nacionales.

En efecto. La conocida como «Quinta Directiva», la Directiva (UE) 2018/843 del Parlamento Europeo y del Consejo de 30 de mayo de 2018 por la que se modi- fica la Directiva (UE) 2015/849 relativa a la Prevención de la Utilización del Sis- tema Financiero para el Blanqueo de Capitales o la Financiación del Terrorismo, y por la que se modifican las Directivas 2009/138/CE y 2013/36/UE15, define las monedas virtuales, en su art. 1, punto 2, letra d), como “representación digital de valor no emitida ni garantizada por un banco central ni por una autoridad pública, no necesariamente asociada a una moneda establecida legalmente, que no posee el estatuto jurídico de moneda o dinero, pero aceptada por personas físicas o jurídicas como medio de cambio y que puede transferirse, almacenarse y negociarse por medios electrónicos".

\subsubsection{Cardano}

Esta es una criptomoneda que carece de mineros, pues opera mediante un nuevo algoritmo denominado "Ouroboros". Este protocolo garantiza la máxima seguridad posible en la 
plataforma. Permite, además, una nueva generación de contratos inteligentes mediante un nuevo lenguaje desarrollado para la utilización del cardano.

Cardano es un proyecto que comenzó en 2015 como un esfuerzo para cambiar la forma en que se diseñan y desarrollan las criptomonedas. El enfoque general, más allá de un conjunto particular de innovaciones, es proporcionar un ecosistema más equilibrado y sostenible que responda mejor a las necesidades de sus usuarios y de futuros sistemas de integración.

Es una red de contrato inteligente que utiliza una arquitectura en capas para enfocarse en la seguridad. Cardano se jacta de ser el primer proyecto de blockchain que se construirá sobre investigación hipotética revisada por pares y se desarrollará a partir de una filosofía lógica.

Cardano se utiliza para enviar y recibir monedas virtuales y es el hogar de la moneda virtual "Ada". Esta moneda digital habla sobre el destino del dinero físico, que influye en intercambios plausibles rápidos $\mathrm{y}$ fáciles de coordinar asegurados mediante la criptografía.

En resumen, Cardano es más que una moneda virtual, es una plataforma innovadora ideal para ejecutar aplicaciones monetarias en el mundo actual.

\subsubsection{Cardano: Escalabilidad, Interoperabilidad y sostenibilidad}

Esta su tercera generación de criptomonedas se define por tres características que mejora y optimiza.

\subsection{Escalabilidad Cardano}

La escalabilidad es una de las cualidades que definen las criptomonedas. Está relacionada con la capacidad de un sistema de adaptarse al crecimiento de trabajo. Por ejemplo, número de transacciones por segundo, a mayor volumen de transacción más volumen de datos a gestionar y la escala de datos (tamaño de block chains debido a transacciones).

La escalabilidad de Cardano es innovadora. En primer lugar, el nuevo algoritmo de extracción Proof-Of-Stake (POS) de Cardano, conocido como Ouroboros. Ouroboros es notable por ser uno de los mecanismos de consenso más eficientes en el espacio de criptomonedas, además de ser el primero en ser probado como seguro.

Así pues, se apuesta por el denominado "Slot Leader", es un nodo con una apuesta positiva que se ha seleccionado para generar un nuevo bloque. La probabilidad de ser elegido se determina en una "lotería justa", donde la cantidad de monedas que posee un nodo corresponde con la probabilidad de ser elegido para generar un nuevo bloque. 
Otro gran factor de Cardano es RINA (Recursive InterNetwork Architecture). RINA es un nuevo tipo de tecnología que pretende reconfigurar la estructura de internet, de RINA puede operar como una red escalable que no requiere que todos los nodos procesen cada transacción.

\subsection{Interoperabilidad Cardano}

Cardano ha convertido la interoperabilidad en uno de sus principales objetivos. En la actualidad, las criptomonedas como Bitcoin, Ethereum y Ripple existen separadas una de la otra y del sistema financiero heredado.

Cardano va un paso más y pretende observar y comprender las transacciones que están sucediendo en múltiples sistemas y blockchains, utilizando su cadena de bloques Cardano y su "token" nativo ADA.

Para ello se vale del sistema Non-Interactive-Proofs-of-Proof-of-Work (NIPoPoWs), capaz de verificar transacciones usando solo una pequeña porción de las cadenas de bloques requeridas. ¡Una auténtica innovación!

\subsection{Sostenibilidad Cardano}

La sostenibilidad es un punto clave para asegurar el futuro del sistema de las criptomonedas. Cardano apuesta por la creación de un "Ministerio de Hacienda" que permite a un blockchain imprimir dinero y almacenarlo en una cuenta bancaria descentralizada.

Probablemente lo más interesante de este concepto es que los interesados pueden hacer propuestas sobre cómo gastar el dinero, utilizando un sistema de votación democrático, dando a todos los interesados la posibilidad de participar en el gobierno y el crecimiento de la cadena de bloques.

Cardano es una plataforma tecnológica innovadora que se esfuerza por convertirse en la tercera generación de criptomonedas y sus propias características definen un sistema fuerte, creativo y con mucho futuro.

\subsubsection{Cotización y Precio de Cardano}

Las criptomonedas son valores muy volátiles, propio delos mercado nuevos y su descentralizada naturaleza. Cardano prácticamente está empezando y no esta todavía asentada como otras competidores suyas.

Veamos un histórico de datos para entender el valor de Cardano:

Diciembre 2017 - 0,719 \$ Enero 2018 - 0,519 \$

Febrero 2018 - 0,312\$ Mayo 2018 - 0,225\$ 
Si bien oscila entre descensos y poco ascenso, para 2019 se prevé que remontará de forma gradual, pues sus sistemas van a comenzar a funcionar $100 \%$. De hecho se estima que para el 2020 obtenga un valor de alrededor $\mathbf{0 , 8 3 7 \$ . ~}$

Es el momento de estudiar, analizar y confiar en el futuro de las criptomonedas llamada Cardano.

\section{Conclusiones}

La articulación entre distintos procesos que antes parecieran inconexos, dado su origen y sus técnicas de análisis escasamente interrelacionados, se convierte ahora, gracias al entorno multisectorial permitido por la sociedad de la información, en una posibilidad viable. Tal es el caso de la confluencia que puede existir entre la teoría de la gestión de la información, la cual tiene sus bases en el análisis cualitativo), y las de la investigación de operaciones (especialmente cadenas de Markov), cuyas técnicas se ajustan más al análisis cuantitativo, desde donde se construye el presente artículo, para mostrar un caso práctico de manejo y transformación de la información, teniendo como epicentro a la criptomoneda bitcoin .

Este recurso criptoactivo, según Oli- veira et al. (2019), amerita rigurosos análisis y estudios que puedan apor- tar a su entendimiento en el marco de los nuevos mercados, las nuevas economías y las nuevas sociedades, intentando predecir, de cierta manera, su comportamiento tan volátil y distinto de los históricamente reconocidos como insumos monetarios.

Como se puede apreciar a lo largo del texto, los resultados expuestos permi- ten evidenciar entonces que es posible combinar ambas técnicas para carac- terizar dicha criptomoneda, determinar su evolución en el mercado, así como pronosticar, de alguna manera, su desempeño futuro. Esto permite brin- dar información válida y oportuna para que sus usuarios tengan un manejo un poco más confiable, desde el punto de vista científico. De esta manera, se pudo establecer que, según las proba- bilidades señaladas, la criptomoneda bitcoin es un recurso realmente esta- ble, e incluso se puede afirmar que, en el largo plazo, seguirá siendo un activo empleado para transferir valor, con una tendencia constante al alza, con un comportamiento semejante, midiendo las proporciones, a lo que ocurre con un activo tan seguro como lo es el oro.

Finalmente, con este artículo se de- muestra que, por más complejo, volátil, emergente, novedoso o moderno que pueda ser un objeto o proceso, este puede estudiarse con las herramientas clásicas de análisis asociadas a la in- formación, máxime ahora que existen 
posibilidades para su complementariedad en tal tipo de ejercicios, como ya ha quedado en evidencia.

\section{Referencias}

Baraona, E., \& Reyes, C. (2018). The weight of bitcoin. Arq, 2018(98), 32-43.

Jimenez, E. R., \& Contreras, J. C. (2018). PROPUESTA DE UN ÍNDICE DE MERCADO PARA CRIPTOMONEDAS. Revista Universitaria Ruta, 2, 49-67.

Https://orcid.org/0000-0003-

Onrubia, J. (2017). El Bitcoin, la nueva moneda virtual y su Ordenamiento jurídico.

Escobar-sarria, J. M. (2014). ¿Sociedad de la información? Miradas sobre la apropiación social de las tecnologías. Cali: Universidad Autónoma de Occidente.

Ziemba, e. (2019). The Contribution of ICT Adoption to the Sustainable Information Society. Journal of Computer Information Systems, 59(2), 116-126.

Vásquez-rizo, f. E., rodríguez-Muñoz, J. V., \& gómez-hernández, J. A. (2019). La gestión de información para medir la capacidad investigadora en una institución de educación superior. Revista Espacios, 40(8), 18-30.

Oliveira-Monteiro, A. H., De souza, A. D., guazzelli-batista, b., \& zaparoli, M. (2019). Market Prediction in Criptocu- rrency: A Systematic Literature Map- ping. Advances in Intelligent Systems and Computing, 800(11), 601-604.

Capps, c. J., cassidy, c. M., gravois, r., \& warner, J. (2019). Expanding the competitive profile matrix: Introducing the production/operations manage- ment, marketing, human resource management, finance/accounting, research and development, and in- formation systems competitive profile matrices. Journal of Business Strate- gies, 36(1), 59-69.

M.; Radia, A.; Thomas, R.: «El dinero en la economía moderna: una introducción», Revista de Economía Institucional, vol. 17, no 33, 2015, pp. 333 y ss. [En línea. Último acceso: marzo de 2019]. Vid. Un resumen del concepto de dinero en Casanueva Cañete, D.; López de la Cruz, N.: «El concepto de criptomoneda y breves consideraciones en torno a su tributación», Documentos- Instituto de Estudios Fiscales, no 10, 2018, pp. 77 y ss.

Pérez López, X.: «Las criptomonedas: consideraciones generales y empleo de las criptomonedas como instrumento de blanqueo de capitales en la Unión Europea y en España», Revista de Derecho Penal y Criminología, no 18, 2017, p. 143. 
Brezo Fernández, F.; Rubio Viñuela, Y.: Bitcoin. La tecnología Blockchain y su investigación, cit., para las cuestiones teóricas, pp. 23 y ss., y para las cuestiones operativas, pp.

63 y ss.

Vid. Nieto Giménez-Montesinos, M.A.; Hernáez Molera, J.: «Monedas virtuales y locales: las paramonedas, ¿nuevas formas de dinero?», Revista de Estabilidad Financiera, no 35, 2018, pp. 103 y ss.; aunque los autores, por cierto, no pueden evitar el permanente recurso a la expresión "moneda virtual".

Banco Central Europeo: Virtual currency schemes -a further analysis, Frankfurt am Main, 2015 [En línea: www.ecb.europa.eu. Último acceso: marzo de 2019]. 